\title{
LIGADURA Y SECCION TUBARIA POR CULDOSCOPIA
}

\section{(Cien casos consecutivos)}

\author{
Dr. Fernando del Corral G., M.D.*
}

La Culdoscopia iniciada por Decker y Cherry en 1944 (1), ha sido un procedimiento de aceptación cada día mayor al cual se le deben notorios avances en el diagnóstico y tratamiento en la Ginecología moderna. Su importancia inicial recidió no solamente en el diagnóstico de enfermedades pélvicas en general, sino en los estudios especiales de infertilidad iniciados por Kelly en 1956 (2) y continuados por Riva y asociados (3) en 1959 y luego por Peretz (4) y colaboradores en 1961. Dos años más tarde Clyman (5) con un nuevo panculdoscopio realizó y publicó varios trabajos $(6,7,8)$, que lo llevaron a introducir la Culdoscopía Operatoria en 1968. Nuestra experiencia en este campo se inició como Culdoscopía diagnóstica en 1964 (10) y sólo hasta 1972 iniciamos la "Culdoscopía Operatoria", para el estudio histológico del ovario en problemas endocrinos y para Ligadura y Sección Tubaria, motivo este último de la siguiente presentación.

\section{Material y Métodos}

Este informe comprende cien culdoscopías operatorias consecutivas orientadas exclusivamente para la Ligadura y Sección de trompas por el procedimiento de Pomeroy. El trabajo fue realizado en su mayoría con

pacientes referidas al servicio de Ginecología del Hospital Universitario, de la Facultad de Medicina, de la Universidad del Valle y sobre un grupo de pacientes privadas del autor que solicitaron voluntariamente dicho procedimiento. Las culdoscopías fueron realizadas en un período de un año, comprendido desde el mes de Mayo de 1972 hasta el mes de Mayo de 1973.

\section{Indicaciones}

Estas se resumen en la Tabla No 1, muchas de estas indicaciones fueron múltiples, presentándose en ocasiones varias de ellas en una sola paciente para ser elegida para el procedimiento. Las indicaciones más frecuentes fueron de índole médica, Tabla № 2, en las cuales se indujeron pacientes con graves padecimientos venosos, antecedentes trombo-embólicos, diabetes, afecciones cardíacas y pulmonares, hipertensión arterial esencial y nefrógena, lupus eritematoso, antecedentes teratogénicos entre las más importantes y otras menos importantes tales como asma bronquial severa, historia de convul-

* Profesor del Departamento de Obstetricia y Ginecología División de Salud - Hospital Universitario del Valle. Universidad del Valle. Cali, Colombia S. A. 
TABLA N: 1

\begin{tabular}{lr} 
Médicas & 55 \\
Psiquiátricas de anovuladores & 5 \\
Intolerancia de & 40 \\
Intolerancia al DIU: & 8 \\
$\quad$ Expulsiones & 26 \\
$\quad$ Hemorragias & 23 \\
Dolor & 6 \\
\hline
\end{tabular}

TABLA No 2

\begin{tabular}{lr}
\hline & Pacientes \\
\hline Terreno varicoso severo & 25 \\
Diabetes & 7 \\
Hipertensión esencial & 6 \\
Hipertensión de origen renal & 5 \\
Enfermedad cardíaca & 3 \\
Enfermedad pulmonar & 2 \\
Antecedentes teratogénicos & 2 \\
Otras & 5 \\
\multicolumn{1}{c}{ TOTAL } & -55 \\
\hline
\end{tabular}

siones, etc... Las causas siquiátricas comprendieron exclusivamente en este grupo cinco pacientes con esquizofrenia crónica indiferenciada. Las otras causas comprendieron de acuerdo a la Tabla N: 1, rechazo a la ingestión de los anovuladores por sus efectos secundarios o a mala tolerancia de los dispositivos intrauterinos 0 a embarazos con dispositivos intrauterinos in situ.

La edad de las pacientes osciló entre 25 y 40 años con un promedio de 34 años.

\section{Paridad}

Todas las pacientes fueron multíparas entre dos (2) y diez (10) hijos, la mayoría, 65 pacientes $(65 \%)$ fueron multíparas con cuatro (4) 0 más hijos. Hubo entre ellas seis (6) pacientes con más de diez (10) hijos.

\section{Técnica}

Previa información cuidadosa del procedimiento a la paciente, esta se hospitaliza la noche anterior donde se practica una preparación quirúrgica completa a abdomen y periné, incluyendo una ducha vaginal y un enema evacuador. A las 21:00 horas se administra una cápsula de Seconal. ${ }^{(R)}$ El día del procedimiento, una hora antes, se administra una cápsula de Seconal; ${ }^{(R)}$ con la paciente en la Sala de Cirugía y a través de una venoclisis se administran 50 mgrs. de Meperidina disuelta en solución salina lentamente, y 5 a 15 mgrs. de Valium buscando la respuesta deseada de relajación de la paciente al medicamento. Colocada la paciente en la posición genu-pectoral deseada mediante un dispositivo especial fabricado por nosotros, se procede a la preparación del campo operatorio por los métodos usuales y se realiza el procedimiento de acuerdo a lo expuesto por Clyman (9). La anestesia fué en su mayoría ( $84 \%$ ) 84 pacientes, local por infiltración del fondo de Dowglas con $5 \mathrm{cc}$. de xilocaína al $2 \%$ con Adrenalina. Los casos restantes, $16(16 \%)$, se utilizó anestesia general por inhalación, debido al rechazo de la paciente a ser colocada en la posición genu-pectoral en estado vigil.

\section{Resultados}

Se practicaron un total de cien culdoscopias operatorias con el objeto de ligar y seccionar las trompas. La duración total del procedimiento osciló entre 8 y 60 minutos con un promedio de 14 minutos. Los resultados se expresan en la Tabla № 3.

Se practicó ligadura y sección bilateral en 96 pacientes. En tres pacientes se hizo ligadura y sección unilateral por los siguientes motivos: 
TABLA N: 3

\begin{tabular}{lr}
\hline & Pacientes \\
\hline Ligadura y sección bilateral & 96 \\
Ligadura y sección unilateral & 3 \\
Falla del procedimiento & 1 \\
\cline { 2 - 2 } TOTAL & 100 \\
\hline
\end{tabular}

una paciente había sido previamente sometida a Salpingectomía por embarazo ectópico, otra, las adherencias tubarias a la pared anterior del abdomen no permitieron el desprendimiento de la trompa y hubo que hacer exploración por vía alta comprobando este hallazgo. En la tercera paciente la ligadura contralateral de la trompa no se pudo realizar por fallas en el sistema de iluminación del instrumento disponible para tal procedimiento y hubo que recurrir a ligar la trompa restante por Colpotomía, sin dificultad. No se pudo practicar el procedimiento en un caso, en el cual la perforación del Douglas fue defectuosa y muy posterior, produciendo sangrado excesivo del peritoneo e inundación a cavidad haciendo imposible la visualización endoscópica. Con esta paciente el sangrado se controló con cierre de la Colpotomía y el procedimiento se realizó por vía suprapúbica.

\section{Comprobación del espécimen por patología}

De las cien Culdoscopias practicadas se envió el espécimen correspondiente a la sección de la trompa, obtenida en 99 pacientes, todas comprobatorias de la presencia histológica de las trompas.

\section{Embarazos}

En el tiempo transcurrido desde el comienzo hasta la realización de este escrito ( 15 meses), se presentó solamente un embarazo en una paciente a la cual se le había practicado cuatro meses atrás el procedimiento. La paciente fué sometida a las 39 semanas a cesárea y se comprobaron los siguientes hallazgos: En un lado la trompa se encontró con la ligadura in situ y en la trompa contra lateral se encontró la ligadura deslizada y en el extremo de la fimbria. Este caso lo hemos considerado como una falla nuestra, más que una falla del procedimiento por sí mismo. Revisando el caso, los especímenes enviados a Patología durante la ligadura y sección, comprobaron la existencia de las trompas.

\section{Tiempo de hospitalización}

El tiempo de permanencia de las pacientes en el Hospital se aprecia en la Tabla No 4, cabe anotar tiempo de hospitalización, que con nuestra experiencia adquirida en la realización de este trabajo, creemos muy probable se pueda reducir a 6 horas el tiempo post-operatorio de observación.

TABLA No 4

\begin{tabular}{rr}
\hline Horas & Casos \\
\hline 6 & 1 \\
24 & 95 \\
36 & 1 \\
748 & 3 \\
\hline
\end{tabular}

\section{Complicaciones}

Se presentaron cuatro casos de morbilidad, dos inmediatos y dos tardíos. Los dos casos de morbilidad inmediata fueron: uno con hemorragia de la cúpula vaginal ya mencionado como falla del procedimiento por perforación defectuosa del Douglas y otro caso con vómito y distensión pos-toperatoria que cedió con reposo y antieméticos antes de 24 horas. Los dos casos de morbilidad tar- 
día fueron debidos a infección y se trató de dos casos de pelvi-peritonitis manejadas satisfactoriamente, una por tratamiento médico y la otra quirúrgicamente. La infección fue debida a falla comprobada en el sistema de esterilización del instrumento.

\section{Sumario}

Se presentan cien (100) casos de Culdoscopia Operatoria dirigida a Ligadura y sección tubaria bilateral como procedimiento de control de la fertilidad. La sección de la trompa se comprobó en todos los casos por estudio histológico. El procedimiento que sólo requirió un promedio de catorce (14) minutos se hizo bajo anestesia local en la mayoría de los casos, $84(84 \%)$.

Sólo se presentó un embarazo probablemente atribuíble a error humano y no a falla del procedimiento.

Las complicaciones y la morbilidad fueron bajas $4 \%$ y posibles de prevenir en lo sucesivo.

\section{Summary}

One hundred (100) cases of Operative "Culdoscopy" directed towards bilateral ligature and section as a procedure for control of fertility are presented. The section of the oviduct was proven in all cases through hystological analysis. The procedure only required an average of fourteen (14) minutes and was accomplished with local anaesthesia in the majority of the cases, 84 ( $84 \%)$.

Only one pregnancy occurred, possibly due to human error and not to failure of the procedure.
Complications and morbility were low, $4 \%$, with possibilities of their prevention in the future.

\section{BIBLIOGRAFIA}

1 DECKER, A., and CHERRY, T.: Culdoscopy a new method in the diagnosis of pelvic disease. Preliminary Report,A m. J. Surg. 64: 40 (1944).

2 KELLY, J. V., and ROCK, J.: Culdoscopy for diagnosis of infertility: Report of 492 cases, Am. J. Obst. \& Gynec. 72: 523 (1956).

3 RIVA, H. L., HATCH, R. P., and BREEN, J. L.: Culdoscopy for infertility. An Analysis of 203 cases, Cit by Clyman M. J. New York State journal of Medicine, Vol. 66, № 14 , July 15, 1966.

4 PERETZ, A., and SHARF, M.: Culdoscopy in Gynecologic diagnosis a review of 404 cases of endoscopic examination, Cit. by Clyman M. J. New York state journal of Medicine Vol. 66, № 14, July 15, 1966.

5 CLYMAN, M. J. A new panculdoscope diagnostic photographic and operative aspects. Obstet. Gynec. 21: 343, 1963.

6 CLYMAN, M. J. A new approach to the study of tubal physiology in situ pacif. Med. Surg. 74: 119, 1966.

7 CLYMAN, M. J. Importance of culdoscopy in fertility studies. New York J. Med. 66: $1867,1966$.

8 CLYMAN, M. J. Silastic hoods in tuboplasty: a new approach to their removal. Fertil, steril 19: $537,1968$.

9 CLYMAN, M. J. Operative Culdoscopy. Obstetrics \& Gynecology. Vol. 32 № 6; 840, 845, Dec. 1968.

10 DEL CORRAL, F., ARTUZ, A. Y GUZMAN, C. A. La culdoscopia como método auxiliar de diagnóstico en Ginecología. Rev. Col. de Obst. y Ginec. XV: 429, 1964. 